\title{
Association of FokI, TaqI, BsmI and ApaI polymorphisms with diabetic retinopathy: a pooled analysis of case-control studies
}

\author{
JingHua Jiao ${ }^{1, * \dagger}$, Yan $\mathrm{Li}^{2, \dagger}$, Shuang $\mathrm{Xu}^{3, \dagger}$, Jingyang $\mathrm{Wu}^{4}$, Song Yue ${ }^{4}$, Lei Liu ${ }^{4 *}$
}

1. Department of Anesthesiology, Central Hospital, Shenyang Medical College, 110024 Shenyang, China.

2. Department of English, School of Fundamental Sciences, China Medical University, 110122 Shenyang, China.

3. Faculty of Medical Library and Information Sciences, China Medical University, 110122, Shenyang, China.

4. Department of Ophthalmology, The First Affiliated Hospital of China Medical University, 110001 Shenyang, China.

† These authors contributed equally to this work and should be considered as co-first authors.

\begin{abstract}
Background: To assess synthetically the association between polymorphisms in the vitamin D receptor (VDR) gene (FokI, BsmI, ApaI, and TaqI) and diabetic retinopathy (DR).

Methods: Pubmed, Embase, ISI Web of Science, Google-scholar and CBMDisc, CNKI and Chongqing VIP databases were searched. A meta-analysis was performed.

Results: Six studies with 636 cases and 1,035 controls were included in this meta-analysis. The outcomes showed that the FokI polymorphism (F allele) of VDR gene had no statistical protective relationship with DR in overall studies. Interestingly, stratification analysis showed that the FokI polymorphism (Fallele) was significantly associated with decreased DR risk in the Chinese population, among included studies without publication bias, during a comparison analysis between normal subjects and DR patients, and among articles published after 2010. However, the TaqI, BsmI and ApaI polymorphisms of VDR gene had no significant association with the risk of DR.

Conclusion: This meta-analysis of case-control studies revealed that the VDR-FokI polymorphism (F allele) decreased the risk of DR in Chinese people, among included studies without publication bias, during a comparison analysis between normal subjects and DR patients, and among articles published after 2010. Further rigorous and prospective studies with large sample size are needed to confirm our findings.
\end{abstract}

Keywords: Diabetic retinopathy; polymorphism; Vitamin D receptor.

DOI: https://dx.doi.org/10.4314/ahs.v18i4.7

Cite as: Jiao J-H, Li Y, Xu S, Wu J, Yue S, Liu L. Association of FokI, TaqI, BsmI and ApaI polymorphisms with diabetic retinopathy: a pooled analysis of case-control studies. Afri Health Sci. 2018;18(4): 891-899. https:// dx.doi.org/10.4314/abs.v18i4.7

\section{Corresponding authors:}

JingHua Jiao, Lei Liu

Address: No. 5 of Nanqi West Road,

TiexiDistrict, Shenyang, 110024, China.

Tel./Fax: +86-13998128639.

E-mail address: jean0905@163.com (J.H. Jiao)

and liuleijiao@163.com (L. Liu).

\section{Introduction}

To date, diabetic retinopathy (DR) is one of most common causes of visual impairment (VI) in adults starting from the age of 20 years until 74 years ${ }^{1}$. According to the reports by the World Health Organization (WHO), there were $4.8 \%$ of the 37 million annual cases of blindness by DR worldwide ${ }^{2}$. A pooled analysis, 1980-2008, revealed that the global prevalence of DR was $34.6 \%$ (95\% confidence interval, CI: $34.5-34.8 \%$ ) in patients with diabetes ${ }^{3}$. 
Although many strategies are performed to prevent $\mathrm{DR}^{4}$, $\mathrm{DR}$ is more prevalent with the increasing prevalence of diabetes mellitus (DM), especially in China ${ }^{5}$. The mechanisms of DR are sophisticated and there are many studies to identify a number of potential susceptibility genes for DR including those coding for vascular endothelial growth factor (VEGF) ${ }^{6}$, angiotensin converting enzyme $(\mathrm{ACE})^{7}$, aldose reductase $(\mathrm{ALR})^{8}$ and receptor for advanced glycation end products (RAGE) .

Furthermore, many studies have shown that Vitamin D plays an important role in calcium metabolism, anti-angiogenic activities and inhibition of proliferation ${ }^{10-12}$. The vitamin D receptor (VDR) is a ligand-dependent transcription factor that in complex with hormonally active Vitamin D, and extensively express in vascular endothelial cells of humans retina ${ }^{13}$. The VDR gene is located on chromosome 12q13-12q14, and it has four frequently single nucleotide polymorphisms (SNPs). These are FokI G/A (rs2228570) change in exon 2, TaqI T/C (rs731236) change in exon 9, BsmI A/G (rs1544410), and ApaI G/T (rs7975232) changes in intron 8. Generally, the FokI polymorphism is located in the start codon, but the BsmI, ApaI and TaqI polymorphisms are located in the 3 ' UTR regions. Therefore, there is an alternative, a transcription initiation site, resulting in a VDR protein with three additional amino acids. A Previous study revealed a novel association between the functional VDR gene (FokI) polymorphism and a risk of DR in patients with Type 1 diabetes $(\mathrm{T} 1 \mathrm{DM})^{14}$. However, another study suggested that FokI polymorphism of VDR gene did not increase risk of DR in Type 2 diabetes (T2DM) in Poland ${ }^{15}$. Hence, previous studies concerning the association between the VDR-FokI gene polymorphism and the risk of DR are limited and rather conflicting. In order to assess the powerful-strength of the association between the VDR gene polymorphism and susceptibility to DR, we conducted this meta-analysis, which would obtain a greater possibility of acquiring or finding reasonably conclusions.

\section{Methods}

\section{Search strategy}

Two independent reviewers (WJY and LL) screened all studies on the relationship between VDR gene polymorphism and DR. Disagreements were dealt with by discussion. If the results of the discussion still had no agreement, another researcher (SY) would make the fi- nal decision. Both English-language databases including Pubmed, Embase, ISI Web of Science, Google (scholar), and Chinese-language databases including China $\mathrm{Na}$ tional Knowledge Infrastructure (CNKI), Chinese Biological Medicine Disc (CBMDisc), Wan Fang (Chinese), Chongqing VIP database were searched using the terms "Vitamin D receptor or VD receptor or VDR", "diabetic retinopathy or DR" and "polymorphism, variant or mutation". The last search was updated on June 10, 2017. Researchers checked reference lists manually to identify additional literatures. Searching was performed without any limits of language, but only English or Chinese articles included.

\section{Inclusion and exclusion criteria of the study} Studies were included based on the following criteria: (1) case-control studies; (2) complete information for VDR gene mutations with the risk of DR for calculating the odds ratio (OR) with 95\% CI directly and indirectly. The exclusion criteria were as follows: (1) editorials, abstracts, case reports, reviews and studies that used an animal or cell line, (2) any data that was insufficient or overlapping, (3) genotypes not in Hardy Weinberg equilibrium (HWE).

\section{Data extraction and quality assessment}

After selection process, data was extracted within a standard form by those two investigators. The following information was extracted: name of the First author, location, publication year, ethnicity, sample sizes and genotype distributions of cases and controls. Newcastle-Ottawa Quality Assessment Scale $(\mathrm{NOS})^{16}$ (Table 1) was used to evaluate the quality of included studies. The score of study with more than 6 stars was considered of relatively high quality.

This meta-analysis of case-control studies was guided and performed by the PRISMA statement ${ }^{17}$ and MOOSE guidelines ${ }^{18}$.

\section{Statistical analysis}

The HWE was conducted for testing the distribution of genotypes in controls within each included study, and a P-value $>0.05$ suggested that the study sample was representative of the population.

The ORs with 95\% CI for allele and genotype models (homozygous model, heterozygous model, recessive model, and dominant model) were used to evaluate the 
association between the VDR gene polymorphisms and the risk of DR. I2 value was used to examine the Heterogeneity among eligible studies. When the heterogeneity was significant $(\mathrm{I} 2>50 \%)$, the random-effects model (based on the DerSimonian and Laird method) would be used to evaluate the pooled ORs, if not, pooled ORs could then be examined by the fixed-effects model (based on the Mantel-Haenszel method). The potential publication bias was assessed with a funnel plot and Egger regression asymmetry test. An asymmetric plot suggested a possible publication bias and the $\mathrm{P}$ value of Egger's test less than 0.05 was considered representative of statistically significant publication bias. If there was publication bias, stratification analysis was carried in order to assess the reason for bias and strength the association between VDRgene polymorphisms and DR. All statistical tests were performed with Stata 11.0 software (StataCorp LP, College Station, TX, USA). All the P-values were two-sided and less than 0.05 were considered to be statistically significant.

\section{Results}

\section{Study characteristics}

After detailed screening the databases, six case-control articles (11 study-samples) ${ }^{14,15,19-22}$ with 636 cases of any DR and 1,035 controls (including DM and normal subjects) were included in this meta-analysis. There were five study-samples researched on the FokI polymorphisms including 535 cases and 936 controls, and two study-samples studied on the BsmI polymorphisms including 179 cases and 408 controls, and two study-samples studied on the ApaI polymorphisms including 179 cases and 408 controls, and two study-samples studied on the TaqI polymorphisms including 186 cases and 281 controls. The selection process of eligible studies was shown in Figure 1. The qualities of the included studies were evaluated by NOS; the quality scores of six included studies were presented in Table 1 . In addition, the characteristics of six articles were shown in Table 2. All of including studies were consistent with HWE. All studies indicated that the controls were DM without DR or non-DM subjects except two articles by Taverna et al. involved non-severe DR subjects as controls ${ }^{14,22}$.

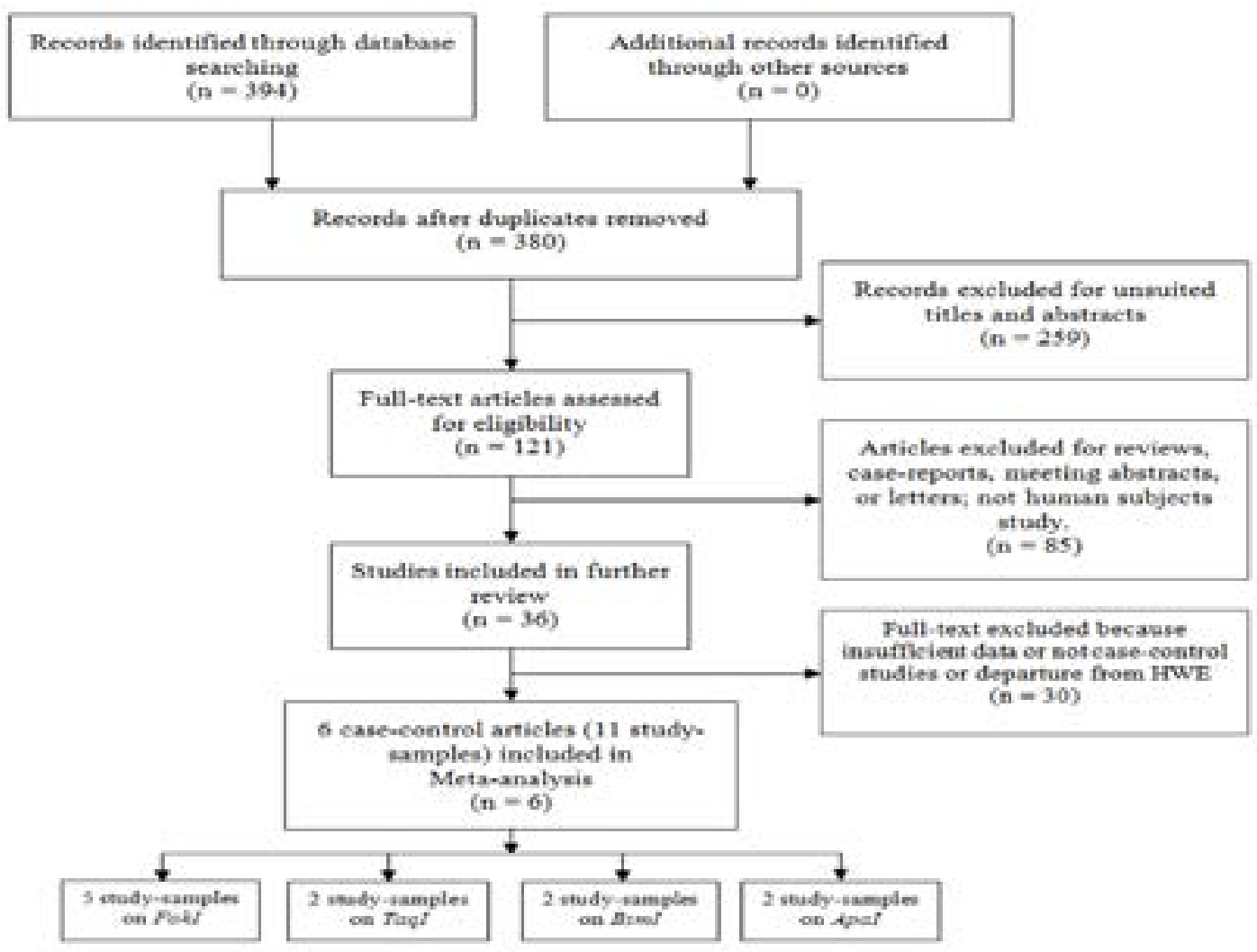

Figure 1. The flow chart of publication selection. 
Table 1. Assessment study quality based on the Newcastle-Ottawa scale.

\begin{tabular}{|c|c|c|c|c|c|c|c|c|c|}
\hline $\begin{array}{c}\text { First } \\
\text { author }\end{array}$ & $\begin{array}{c}\text { Is the } \\
\text { case } \\
\text { definition } \\
\text { adequate } \\
\text { ? }\end{array}$ & $\begin{array}{l}\text { Representativenes } \\
\text { s of the cases }\end{array}$ & $\begin{array}{c}\text { Selectio } \\
\text { n of } \\
\text { controls }\end{array}$ & $\begin{array}{c}\text { Definitio } \\
n \text { of } \\
\text { controls }\end{array}$ & $\begin{array}{l}\text { Comparabilit } \\
y \text { of cases and } \\
\text { controls on } \\
\text { the basis of } \\
\text { the design or } \\
\text { analysis }\end{array}$ & $\begin{array}{l}\text { Ascertainmen } \\
\text { t of exposure }\end{array}$ & $\begin{array}{c}\text { Same method } \\
\text { of } \\
\text { ascertainmen } \\
t \text { for } \\
\text { cases and } \\
\text { controls }\end{array}$ & $\begin{array}{l}\text { Non- } \\
\text { respons } \\
\text { e rate }\end{array}$ & $\begin{array}{c}\text { Tota } \\
1 \\
\text { score }\end{array}$ \\
\hline Taverna $^{[14]}$ & $\star$ & मे & 古 & $\star$ & $\star \star$ & $\star$ & $\star$ & मे & 6 \\
\hline Cyganek $_{]}^{[15}$ & $\star$ & $\star$ & $\star$ & $\star$ & $\star \star$ & $\star$ & $\star$ & मे & 8 \\
\hline $\mathrm{Hou}^{[20]}$ & $\star$ & $\star$ & 战 & $\star$ & 太放 & $\star$ & $\star$ & मे & 6 \\
\hline Zhong $^{[21]}$ & $\star$ & $\star$ & मे & $\star$ & 太的 & $\star$ & $\star$ & 直 & 6 \\
\hline Taverna $^{[22]}$ & $\star$ & $\star$ & it & $\star$ & 太放 & $\star$ & $\star$ & it & 6 \\
\hline
\end{tabular}

Table 2. Characteristics of studies on DR and VDR polymorphism.

\begin{tabular}{|c|c|c|c|c|c|c|c|c|c|c|c|c|c|c|c|c|c|c|c|c|c|c|}
\hline \multirow{2}{*}{$\begin{array}{c}\text { Author } \\
\text { Fokl }\end{array}$} & \multirow[t]{2}{*}{ Year } & \multirow[t]{2}{*}{ Ethnicity } & \multirow[t]{2}{*}{$\begin{array}{l}\text { Genotyping } \\
\text { Methods }\end{array}$} & \multirow[t]{2}{*}{ Control Types } & \multirow[t]{2}{*}{$\begin{array}{l}\text { Type of } \\
\text { diabetes }\end{array}$} & \multicolumn{2}{|c|}{$\mathrm{n}$ (control) } & \multirow[t]{2}{*}{ DR grade } & \multicolumn{2}{|c|}{$\mathrm{n}$ (case) } & \multirow[t]{2}{*}{$\begin{array}{l}\text { Duration of DM } \\
\text { (case/control, year) }\end{array}$} & \multicolumn{5}{|c|}{ Case } & \multicolumn{5}{|c|}{ Control } & \multirow[t]{2}{*}{ HWE } \\
\hline & & & & & & Male & Female & & Male & Female & & FF & Ff & $\mathrm{ff}$ & F & $\mathrm{f}$ & $\mathrm{FF}$ & Ff & $\mathrm{ff}$ & F & $\mathrm{f}$ & \\
\hline$W_{u}\left[{ }^{[!]}\right]$ & 2010 & Chinese & PCR-RFLP & normal & T2DM & 32 & 30 & Any DR & 30 & 38 & $\mathrm{~N} / \mathrm{A}$ & 6 & 37 & 19 & 49 & 75 & 21 & 35 & 12 & 77 & 59 & 0.69 \\
\hline \multirow[t]{2}{*}{ Houl2y } & 2015 & Chinese & PCR-RFLP & normal & T2DM & 58 & 62 & NPDR & 40 & 40 & $\mathrm{~N} / \mathrm{A}$ & 5 & 20 & 55 & 30 & 130 & 14 & 50 & 56 & 78 & 162 & 0.58 \\
\hline & & & & DM & & 45 & 47 & PDR & 40 & 42 & & 5 & 20 & 57 & 30 & 134 & 12 & 34 & 46 & 58 & 126 & 0.16 \\
\hline \multirow[t]{2}{*}{ Zhonge[2] } & 2015 & Chinese & PCR-RFLP & DM & T2DM & 51 & 59 & Any DR & 44 & 50 & $10.0 / 5.0$ & 11 & 53 & 30 & 75 & 113 & 35 & 61 & 14 & 131 & 89 & 0.11 \\
\hline & & & & normal & & 54 & 62 & & & & & $\mathrm{~N} / \mathrm{A}$ & $\mathrm{N} / \mathrm{A}$ & $\mathrm{N} / \mathrm{A}$ & $\mathrm{N} / \mathrm{A}$ & $\mathrm{N} / \mathrm{A}$ & 40 & 58 & 18 & 138 & 94 & 0.68 \\
\hline Cyganek $k^{[15]}$ & 2006 & Caucasians & PCR-RFLP & DM & T2DM & 85 & 97 & Any DR & 36 & 49 & $14.6 / 9.7$ & 21 & 43 & 21 & 85 & 85 & 51 & 93 & 38 & 195 & 169 & 0.7 \\
\hline Taverna ${ }^{[1]]}$ & 2005 & Caucasians & PCR-RFLP & $\begin{array}{l}\text { Non-severe } \\
\text { DR group }\end{array}$ & TIDM & 127 & 127 & severe DR & 59 & 67 & $25.5 / 25.1$ & 38 & 65 & 23 & 111 & 141 & 15 & 56 & 57 & 86 & 170 & 0.82 \\
\hline TaqI & & & & & & & & & & & & TT & $\mathrm{Tt}$ & $\pi t$ & $\mathrm{~T}$ & $t$ & TT & $\mathrm{Tt}$ & $\mathrm{tt}$ & $\mathrm{T}$ & $\mathrm{t}$ & \\
\hline $\operatorname{Taverna}^{[2]}$ & 2002 & Caucasians & PCR-RFLP & $\begin{array}{c}\text { Non-severe } \\
\text { DR } \\
\text { group/normal }\end{array}$ & TIDM & 58 & 41 & severe DR & 45 & 56 & $30.0 / 25.2$ & 27 & 58 & 16 & 112 & 90 & 42 & 44 & 13 & 128 & 70 & 0.78 \\
\hline Cyganek ${ }^{[15]}$ & 2006 & Caucasians & PCR-RFLP & DM & T2DM & 85 & 97 & Any DR & 36 & 49 & $14.6 / 9.7$ & 40 & 38 & 7 & 118 & 52 & 82 & 82 & 18 & 246 & 118 & 0.7 \\
\hline BsmI & & & & & & & & & & & & GG & $\mathrm{GA}$ & $\mathrm{AA}$ & $G$ & $\mathrm{~A}$ & GG & $\mathrm{GA}$ & $\mathrm{AA}$ & $G$ & $\mathrm{~A}$ & \\
\hline \multirow[t]{2}{*}{ Zhongh[s] } & 2015 & Chinese & PCR-RFLP & DM & T2DM & 51 & 59 & Any DR & 44 & 50 & $10.0 / 5.0$ & 5 & 27 & 62 & 37 & 151 & 6 & 27 & 77 & 39 & 181 & 0.09 \\
\hline & & & & normal & & 54 & 62 & & $\mathrm{~N} / \mathrm{A}$ & $\mathrm{N} / \mathrm{A}$ & & $\mathrm{N} / \mathrm{A}$ & $\mathrm{N} / \mathrm{A}$ & $\mathrm{N} / \mathrm{A}$ & $\mathrm{N} / \mathrm{A}$ & $\mathrm{N} / \mathrm{A}$ & 2 & 18 & 96 & 22 & 210 & 0.3 \\
\hline \multirow{2}{*}{$\begin{array}{c}\text { Cyganekk[1] } \\
\text { Apal }\end{array}$} & 2006 & Caucasians & PCR-RFLP & DM & T2DM & 85 & 97 & Any DR & 36 & 49 & $14.6 / 9.7$ & 10 & 37 & 38 & 57 & 113 & 21 & 84 & 77 & 126 & 238 & 0.79 \\
\hline & & & & & & & & & & & & $\mathrm{AA}$ & $\mathrm{AC}$ & $\mathrm{CC}$ & $\mathrm{A}$ & $\mathrm{C}$ & $\mathrm{AA}$ & $\mathrm{AC}$ & $\mathrm{CC}$ & $\mathrm{A}$ & $\mathrm{C}$ & \\
\hline \multirow[t]{2}{*}{ Zhongh[2] } & 2015 & Chinese & PCR-RFLP & DM & T2DM & 51 & 59 & Any DR & 44 & 50 & $10.0 / 5.0$ & 27 & 54 & 13 & 108 & 80 & 34 & 60 & 16 & 128 & 92 & 0.2 \\
\hline & & & & normal & & 54 & 62 & & $\mathrm{~N} / \mathrm{A}$ & $\mathrm{N} / \mathrm{A}$ & & $\mathrm{N} / \mathrm{A}$ & $\mathrm{N} / \mathrm{A}$ & $\mathrm{N} / \mathrm{A}$ & $\mathrm{N} / \mathrm{A}$ & $\mathrm{N} / \mathrm{A}$ & 29 & 59 & 28 & 117 & 115 & 0.85 \\
\hline Cyganek $[1[1]$ & 2006 & Caucasians & PCR-RFLP & DM & T2DM & 85 & 97 & Any DR & 36 & 49 & $14.6 / 9.7$ & 17 & 39 & 29 & 73 & 97 & 39 & 100 & 43 & 178 & 186 & 0.17 \\
\hline
\end{tabular}

Abbreviation: T2DM, type 2 diabetes mellitus; TIDM, type 1 diabetes mellitus; DR, diabetic retinopathy; N/A: Not Avalable. PCR-RFLP: Polymerase chain reaction-restriction fragment length polymorphism; HWE: Hardy Weinberg equilibrium; NPDR: non-proliferative diabetic retinopathy; PDR: proliferative diabetic 


\section{Meta-analysis}

Overall, there was no link between VDR-FokI polymorphism and DR [F vs. f: OR $=0.68$ (95\% CI: 0.42-1.11); FF vs. ff: OR $=0.55$ (95\% CI: 0.14-2.11); Ff vs. ff: OR = 0.79, (95\% CI: 0.39-1.62); FF vs. Ff+ff: OR $=0.62(95 \%$ CI: $0.24-1.59)$; FF+Ff vs. ff: OR $=0.73$ (95\% CI: $0.31-$ 1.72)]. In stratification analysis, the $F$ allele in FokIpolymorphism lowered the risk of DR in Chinese people [F vs. f: $\mathrm{OR}=0.47(95 \% \mathrm{CI}$ : 0.38-0.58); FF vs. Ff: OR = 0.23 (95\%CI: 0.14-0.38); Ff vs. ff: OR $=0.48$ (95\%CI: 0.34-0.67); FF vs. Ff+ff: OR = 0.52 (95\%CI: 0.33-0.83); FF+Ff vs. ff: $\mathrm{OR}=0.41$ (95\%CI: 0.29-0.56)], comparison between DR patients and non-DM subjects as controls [F vs. f: $\mathrm{OR}=0.47$ (95\%CI: 0.37-0.59); FF vs. Ff: $\mathrm{OR}=$
0.23 (95\%CI: 0.13-0.39); Ff vs. ff: OR $=0.48$ (95\%CI: 0.33-0.71); FF vs. Ff+ff: $\mathrm{OR}=0.31$ (95\%CI: 0.19-0.5); $\mathrm{FF}+\mathrm{Ff}$ vs. ff: $\mathrm{OR}=0.41$ (95\%CI: 0.28-0.58)], and studies published after 2010 [F vs. f: OR $=0.47$ (95\%CI: 0.38-0.58); $\mathrm{FF}$ vs. Ff: $\mathrm{OR}=0.23$ (95\%CI: 0.14-0.38); Ff vs. ff: $\mathrm{OR}=0.48$ (95\%CI: 0.34-0.67); FF vs. Ff+ff: $\mathrm{OR}=0.52$ (95\%CI: 0.33-0.83); FF+Ff vs. ff: $\mathrm{OR}=0.41$ (95\%CI: 0.29-0.56)], respectively. Moreover, the $\mathrm{F}$ allele in FokIpolymorphisms of VDR gene had a protective effect on the risk of DR when the study taken non-severe $\mathrm{DR}$ subjects as controls was excluded [F vs. f: $\mathrm{OR}=$ 0.56 (95\%CI: 0.41-0.76); FF vs. Ff: OR $=0.31$ (95\%CI: 0.15-0.64); Ff vs. ff: OR = 0.54 (95\%CI: 0.4-0.72); FF vs. Ff+ff: OR $=0.42(95 \% \mathrm{CI}: 0.23-0.77) ; \mathrm{FF}+\mathrm{Ff}$ vs. ff: OR $=0.47$ (95\%CI: 0.35-0.62), Table 3 .

Table 3. Pooled ORs and $95 \%$ CIs of overall and stratification analysis for association between DR and FokI polymorphism.

\begin{tabular}{|c|c|c|c|c|c|c|c|c|c|c|c|c|c|c|c|c|c|c|}
\hline FokI & $\begin{array}{c}\text { Study } \\
(\mathrm{n})\end{array}$ & Case & Control & $\mathrm{F} / \mathrm{f}$ & $\begin{array}{r}\text { Effects-model } \\
\text { (heterogeneity) }\end{array}$ & $P$ & $\mathrm{FF} / \mathrm{ff}$ & $\begin{array}{r}\text { Effects-model } \\
\text { (heterogeneity) }\end{array}$ & $P$ & $\mathrm{Ff} / \mathrm{ff}$ & $\begin{array}{r}\text { Effects-model } \\
\text { (heterogeneity) }\end{array}$ & $\mathrm{P}$ & $\mathrm{FF} / \mathrm{Ff}+\mathrm{ff}$ & $\begin{array}{r}\text { Effects-model } \\
\text { (heterogeneity) }\end{array}$ & $\mathrm{P}$ & $\mathrm{FF}+\mathrm{Ff} / \mathrm{ff}$ & $\begin{array}{r}\text { Effects-model } \\
\text { (heterogeneity) }\end{array}$ & $\mathrm{P}$ \\
\hline Total & 5 & 535 & 936 & $\begin{array}{c}0.68 \\
(0.42- \\
1.11)\end{array}$ & $\mathrm{R}(87.8 \%)$ & 0.12 & $\begin{array}{c}0.55 \\
(0.14- \\
2.11)\end{array}$ & $\mathrm{R}(92.4 \%)$ & 0.38 & $\begin{array}{c}0.79 \\
(0.39- \\
1.62)\end{array}$ & $\mathrm{R}(85.2 \%)$ & 0.53 & $\begin{array}{c}0.62 \\
(0.24- \\
1.59)\end{array}$ & $\mathrm{R}(88.4 \%)$ & 0.32 & $\begin{array}{c}0.73 \\
(0.31- \\
1.72)\end{array}$ & $\mathrm{R}(90.9 \%)$ & 0.47 \\
\hline $\begin{array}{l}\text { Without publication } \\
\text { bias }\end{array}$ & 4 & 409 & 682 & $\begin{array}{c}0.56 \\
(0.41- \\
0.76)\end{array}$ & $\mathrm{R}(62.1 \%)$ & $<0.01$ & $\begin{array}{c}0.31 \\
(0.15- \\
0.64)\end{array}$ & $\mathrm{R}(66.9 \%)$ & 0.002 & $\begin{array}{l}0.54 \\
(0.4- \\
0.72)\end{array}$ & $\mathrm{F}(1.7 \%)$ & $<0.01$ & $\begin{array}{c}0.42 \\
(0.23- \\
0.77)\end{array}$ & $\mathrm{R}(63.3 \%)$ & 0.005 & $\begin{array}{c}0.47 \\
(0.35- \\
0.62)\end{array}$ & $\mathrm{F}(30.4 \%)$ & $<0.01$ \\
\hline Chinese & 3 & 324 & 500 & $\begin{array}{c}0.47 \\
(0.38- \\
0.58)\end{array}$ & $\mathrm{F}(0)$ & $<0.01$ & $\begin{array}{c}0.23 \\
(0.14- \\
0.38)\end{array}$ & $\mathrm{F}(7.8 \%)$ & $<0.01$ & $\begin{array}{r}0.48 \\
(0.34- \\
0.67)\end{array}$ & $\mathrm{F}(0)$ & $<0.01$ & $\begin{array}{c}0.52 \\
(0.33- \\
0.83)\end{array}$ & $\mathrm{F}(2.3 \%)$ & 0.006 & $\begin{array}{c}0.41 \\
(0.29- \\
0.56)\end{array}$ & $\mathrm{F}(0)$ & $<0.01$ \\
\hline Caucasians & 2 & 211 & 436 & $\begin{array}{c}1.16 \\
(0.65- \\
2.06)\end{array}$ & $\mathrm{R}(80.1 \%)$ & 0.61 & $\begin{array}{c}2.15 \\
(0.26- \\
17.41)\end{array}$ & $\mathrm{R}(93.5 \%)$ & 0.47 & $\begin{array}{r}1.56 \\
(0.46- \\
5.23)\end{array}$ & $\mathrm{R}(86.7 \%)$ & 0.47 & $\begin{array}{c}1.22 \\
(0.78- \\
1.89)\end{array}$ & $\mathrm{F}(48.6 \%)$ & 0.35 & $\begin{array}{c}1.71 \\
(0.39- \\
7.41)\end{array}$ & $\mathrm{R}(91.9 \%)$ & 0.47 \\
\hline DR vs. DM & 4 & 467 & 638 & $\begin{array}{c}0.74 \\
(0.42- \\
1.32)\end{array}$ & $\mathrm{R}(88.6 \%)$ & 0.31 & $\begin{array}{c}0.71 \\
(0.14- \\
3.44)\end{array}$ & $\mathrm{R}(93.1 \%)$ & 0.66 & $\begin{array}{c}0.84 \\
(0.35- \\
2.03)\end{array}$ & $\mathrm{R}(87.2 \%)$ & 0.69 & $\begin{array}{c}0.78 \\
(0.27- \\
2.22)\end{array}$ & $\mathrm{R}(88.6 \%)$ & 0.64 & $\begin{array}{c}0.8 \\
(0.27- \\
2.33)\end{array}$ & $\mathrm{R}(92.1 \%)$ & 0.68 \\
\hline DR vs. T2DM & 2 & 179 & 292 & $\begin{array}{c}0.63 \\
(0.33- \\
1.19)\end{array}$ & $\mathrm{R}(82.3 \%)$ & 0.15 & $\begin{array}{c}0.34 \\
(0.07- \\
1.67)\end{array}$ & $\mathrm{R}(86.2 \%$ & 0.18 & $\begin{array}{c}0.59 \\
(0.29- \\
1.21)\end{array}$ & $\mathrm{R}(52.8 \%)$ & 0.15 & $\begin{array}{c}0.5 \\
(0.17- \\
1.45)\end{array}$ & $\mathrm{R}(80.1 \%)$ & 0.21 & $\begin{array}{l}0.51 \\
(0.2- \\
1.29)\end{array}$ & $\mathrm{R}(74.8 \%)$ & 0.15 \\
\hline DR vs. Normal & 3 & 304 & 298 & $\begin{array}{c}0.47 \\
(0.37- \\
0.59)\end{array}$ & $\mathrm{F}(0)$ & $<0.01$ & $\begin{array}{c}0.23 \\
(0.13- \\
0.39)\end{array}$ & $\mathrm{F}(0)$ & $<0.01$ & $\begin{array}{c}0.48 \\
(0.33- \\
0.71)\end{array}$ & $\mathrm{F}(0)$ & $<0.01$ & $\begin{array}{c}0.31 \\
(0.19- \\
0.5)\end{array}$ & $\mathrm{F}(0)$ & $<0.01$ & $\begin{array}{c}0.41 \\
(0.28- \\
0.58)\end{array}$ & $\mathrm{F}(0)$ & $<0.01$ \\
\hline Published after 2010 & 3 & 324 & 500 & $\begin{array}{c}0.47 \\
(0.38- \\
0.58)\end{array}$ & $\mathrm{F}(0)$ & $<0.01$ & $\begin{array}{c}0.23 \\
(0.14- \\
0.38)\end{array}$ & $\mathrm{F}(7.8 \%)$ & $<0.01$ & $\begin{array}{r}0.48 \\
(0.34- \\
0.67)\end{array}$ & $\mathrm{F}(0)$ & $<0.01$ & $\begin{array}{c}0.52 \\
(0.33- \\
0.83)\end{array}$ & $\mathrm{F}(2.3 \%)$ & 0.006 & $\begin{array}{c}0.41 \\
(0.29- \\
0.56)\end{array}$ & $\mathrm{F}(0)$ & $<0.01$ \\
\hline $\begin{array}{l}\text { Published before } \\
2010\end{array}$ & 2 & 211 & 436 & $\begin{array}{c}1.16 \\
(0.65- \\
2.06)\end{array}$ & $\mathrm{R}(80.1 \%)$ & 0.61 & $\begin{array}{c}2.15 \\
(0.26- \\
17.41)\end{array}$ & $\mathrm{R}(93.5 \%)$ & 0.47 & $\begin{array}{c}1.56 \\
(0.46- \\
5.23)\end{array}$ & $\mathrm{R}(86.7 \%)$ & 0.47 & $\begin{array}{c}1.22 \\
(0.78- \\
1.89)\end{array}$ & $\mathrm{F}(48.6 \%)$ & 0.35 & $\begin{array}{c}1.71 \\
(0.39- \\
7.41)\end{array}$ & R (91.9\%) & 0.47 \\
\hline
\end{tabular}

The results in Table 4, Table 5 and Table 6 indicated that there was no statistical significant association between polymorphisms in the VDR gene including BsmI, TaqI, ApaI and the risk of DR. 
Table 4. Pooled ORs and 95\% CIs of analysis for association between DR and ApaI polymorphism.

\begin{tabular}{|c|c|c|c|c|c|c|c|c|c|c|c|c|c|c|}
\hline Gene study case control & $1 \mathrm{~A} / \mathrm{C}$ & $\begin{array}{c}\text { Effects- } \\
\text { model(heterogeneity) }\end{array}$ & $P$ & $\mathrm{AA} / \mathrm{CC}$ & $\begin{array}{l}\text { Effects- } \\
\text { model(heterogeneity) }\end{array}$ & $\mathrm{P} \quad \mathrm{AC} / \mathrm{CC}$ & $\begin{array}{l}\text { Effects- } \\
\text { model(heterogeneity) }\end{array}$ & $\mathrm{PA}$ & $\mathrm{AA} / \mathrm{AC}+\mathrm{CC}_{\mathrm{r}}$ & $\begin{array}{c}\text { Effects- } \\
\text { model(heterogeneity) }\end{array}$ & $\mathrm{P}$ & $\mathrm{AA}+\mathrm{AC} / \mathrm{CC}$ & $\begin{array}{l}\text { Effects- } \\
\text { model(heterogeneity) }\end{array}$ & $P$ \\
\hline 179408 & $\begin{array}{c}0.95 \\
(0.66- \\
1.37)\end{array}$ & $\mathrm{R}(52.5 \%)$ & & $\begin{array}{r}0.96 \\
9(0.44- \\
2.12)\end{array}$ & $\mathrm{R}(54.9 \%)$ & $\begin{array}{cc} & 0.93 \\
0.92 & (0.36- \\
& 2.41)\end{array}$ & $\mathrm{R}(77 \%)$ & 0.88 & $\begin{array}{c}0.98(0.66- \\
1.49)\end{array}$ & $\mathrm{F}(0)$ & 0.96 & $\begin{array}{c}0.93(0.38- \\
2.31)\end{array}$ & $\mathrm{R}(76.6 \%)$ & 0.88 \\
\hline
\end{tabular}

Table 5. Pooled ORs and 95\% CIs of analysis for association between DR and BsmI polymorphism.

\begin{tabular}{|c|c|c|c|c|c|c|c|c|c|c|c|c|c|c|c|c|c|c|}
\hline Gene & study & case & control & $\mathrm{G} / \mathrm{A}$ & $\begin{array}{l}\text { Effects-model } \\
\text { (heterogeneity) }\end{array}$ & $\mathrm{P}$ & $\mathrm{GG} / \mathrm{AA}$ & $\begin{array}{l}\text { Effects-model } \\
\text { (heterogeneity) }\end{array}$ & $\mathrm{P}$ & GA/AA & $\begin{array}{l}\text { Effects-model } \\
\text { (heterogeneity) }\end{array}$ & $P$ & $\mathrm{GG} / \mathrm{GA}+\mathrm{AA}$ & $\begin{array}{l}\text { Effects-model } \\
\text { (heterogeneity) }\end{array}$ & $P$ & $\mathrm{GG}+\mathrm{GA} / \mathrm{AA}$ & $\begin{array}{l}\text { Effects-model } \\
\text { (heterogeneity) }\end{array}$ & $\mathrm{P}$ \\
\hline BsmI & 2 & 179 & 408 & $\begin{array}{c}1.21 \\
(0.74- \\
1.97)\end{array}$ & $\mathrm{R}(63.5 \%)$ & 0.45 & $\begin{array}{l}1.19 \\
(0.6- \\
2.35)\end{array}$ & $\mathrm{F}(0)$ & 0.62 & $\begin{array}{c}1.22 \\
(0.66- \\
2.26)\end{array}$ & R (59.7\%) & 0.53 & $\begin{array}{l}0.8(0.26- \\
2.44)\end{array}$ & $\mathrm{R}(60.6 \%)$ & 0.7 & $\begin{array}{c}1.23(0.67- \\
2.26)\end{array}$ & $\mathrm{R}(62.9 \%)$ & 0.49 \\
\hline
\end{tabular}

Abbreviation: R, random effects-model; F, fixed-effects-model; OR, odds ratio; CI, confidence interval; DR, diabetic retinopathy.

Table 6. Pooled ORs and 95\% CIs of analysis for association between DR and TaqI polymorphism.

\begin{tabular}{|c|c|c|c|c|c|c|c|c|c|c|c|c|c|c|c|}
\hline Gene study case control & $\mathrm{T} / \mathrm{t}$ & $\begin{array}{c}\text { Effects- } \\
\text { model(heterogeneity) }\end{array}$ & $P$ & $\mathrm{TT} / \mathrm{tt}$ & $\begin{array}{c}\text { Effects- } \\
\text { model(heterogeneity) }\end{array}$ & $P$ & $\mathrm{Tt} / \mathrm{tt}$ & $\begin{array}{c}\text { Effects- } \\
\text { model(heterogeneity) }\end{array}$ & $\mathrm{P}$ & $\mathrm{TT} / \mathrm{Tt}+\mathrm{tt}$ & $\begin{array}{c}\text { Effects- } \\
\text { model(heterogeneity) }\end{array}$ & $\mathrm{P}$ & $\mathrm{TT}+\mathrm{T} t / \mathrm{tt}$ & $\begin{array}{c}\text { Effects- } \\
\text { model(heterogeneity) }\end{array}$ & $\mathrm{P}$ \\
\hline $\begin{array}{llll}\text { TaqI } & 2 & 186 & 281\end{array}$ & $\begin{array}{c}0.87 \\
(0.54- \\
1.36)\end{array}$ & $\mathrm{R}(62.6 \%)$ & 0.53 & $\begin{array}{r}0.79 \\
(0.33- \\
1.87)\end{array}$ & $\mathrm{R}(43.2 \%)$ & 0.59 & $\begin{array}{l}1.12(0.6- \\
2.09)\end{array}$ & $\mathrm{F}(0)$ & 0.19 & $\begin{array}{c}0.74 \\
(0.34-1.6)\end{array}$ & $\mathrm{R}(73.7 \%)$ & 0.45 & $\begin{array}{c}0.96 \\
(0.53- \\
1.74)\end{array}$ & $\mathrm{F}(0)$ & 0.9 \\
\hline
\end{tabular}

Abbreviation: R, random effects-model; F, fixed-effects-model; OR, odds ratio; CI, confidence interval; DR, diabetic retinopathy.

\section{Publication bias}

The potential publication bias was examined by using a funnel plot for the allele $\mathrm{F}$ vs. $\mathrm{f}$ against the reciprocal of its standard error (Figure 2). Egger`s test provided con- sistent results in the overall $(\mathrm{t}=-0.22, \mathrm{P}<0.05)$; When the study in which control group including non-severe DR patients was excluded, there was no significant publication bias according to funnel plot (Figure 3).

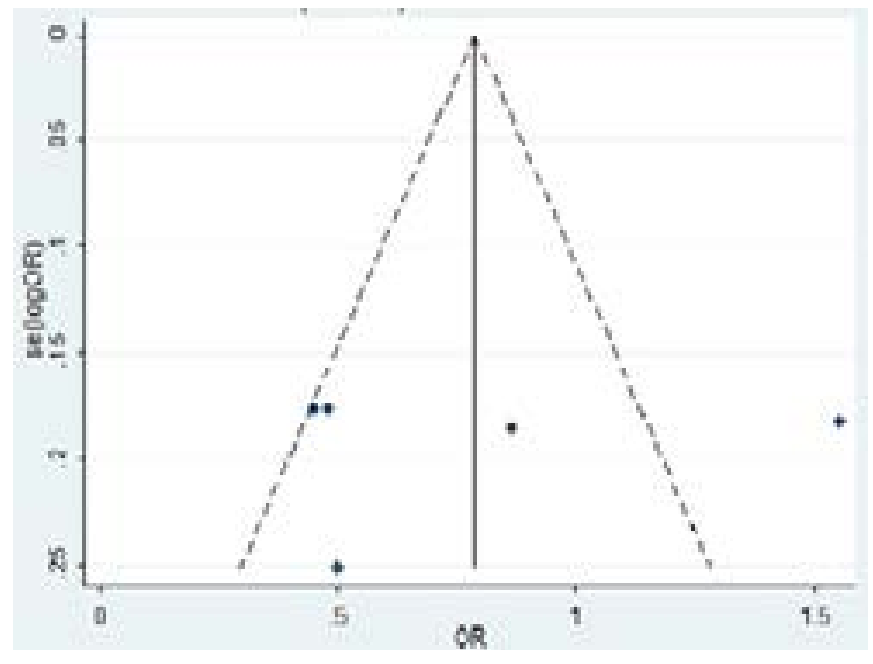

Figure 2. The funnel plot with pseudo $95 \%$ confidence interval of all the evaluable publications for FokI polymorphism ( $F v s$. $f$ allele). 


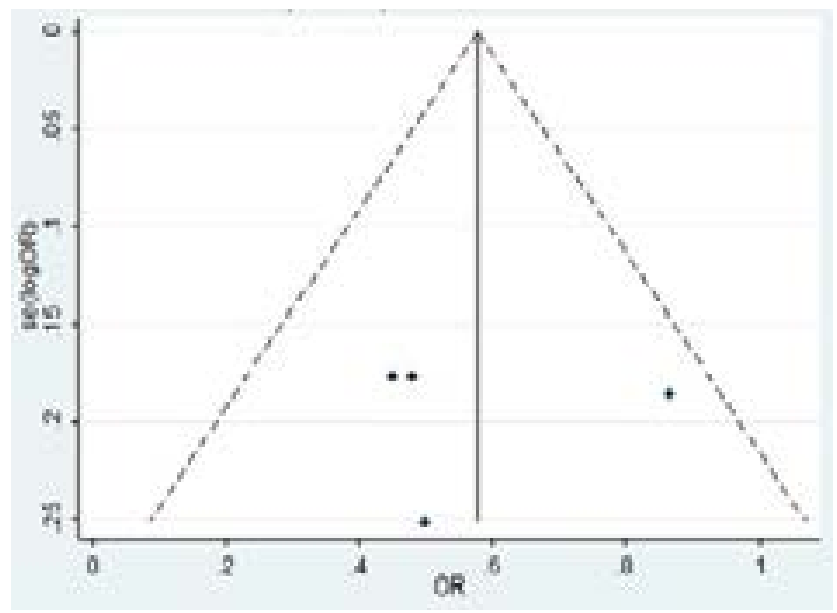

Figure 3. The funnel plot with pseudo $95 \%$ confidence interval of the evaluable publications except for study which can effect on publication bias.

\section{Discussion}

We performed a meta-analysis of case-control studies to evaluate the association between VDR gene polymorphisms and the risk of DR. To the best of our knowledge, Vitamin D supplements will lower the risk of DR in DM patients ${ }^{23}$. However, metabolic activation of vitamin $\mathrm{D}$ is mediated through binding to a VDR. Therefore, the expression of the VDR gene might be involved in the pathogenesis of DR. There were some studies conducted on VDR gene polymorphisms that had been investigated and shown to be associated with presence of any $\mathrm{DR}^{24,25}$. However, the published results were not consistent. Meta-analysis is needed to strengthen the link between VDR gene polymorphisms and DR.

VDR gene is located in chromosomes $12 \mathrm{q} 13-12 \mathrm{q} 14^{26}$, and the allele of FokI polymorphism is located in the 5 end of the VDR gene. Overall, there was no significant association between VDR-FokI polymorphism and risk of DR in this meta-analysis. Notably, we detected a significantly ( $F$ allele) decreased susceptibility for DR among Chinese population, cases with DR compared with normal controls, among studies without publication bias and articles published after 2010 year for all models. In contrast, there was no significant association that was found in Caucasians, cases with DR compared with DM controls, and even cases with DR compared with T2DM controls. Another meta-analysis revealed that FokI polymorphism was possibly a risk factor for T2DM, mostly in Chinese subjects ${ }^{27}$. However, it was inexplicable that $\mathrm{F}$ allele within FokI polymorphism lowered risk for DR in normal subjects rather than DM or T2DM.

BsmI, ApaI and TaqI are the other three polymorphisms for VDR gene. In our meta-analysis, there was no significant association between these three polymorphisms and DR, which may be due to different locations of VDR gene on its chromosome. These three polymorphisms are located in the $3^{\prime}$ UTR regions of VDR gene, but another single-nucleotide polymorphism (FokI) located in the start codon of VDR gene.

According to the results, there was significant heterogeneity detection in some genetic models. Although we have carried out stratification analysis, significant heterogeneity was also detected. After reviewing all included studies, different ethnicity and control subjects may contribute to the heterogeneity. Therefore, the conclusions should be interpreted with caution.

Another previous meta-analysis ${ }^{28}$ revealed that DR was significantly associated with VDR- FokI polymorphism both in overall and sub-group analysis which was not consistent with our results. Zhang et al. study has some limitations. There were seven studies included in that meta-analysis but lacking of the sub-groups analysis stratified by control population (diabetic and normal subjects) or publication date. Moreover, there is lacking of detailed description on study design in the research by Bućan $\mathrm{K}$ et al. ${ }^{25}$ which was also included in the Zhang`s meta-anal- 
ysis. So we excluded this study and only six case-control studies were included in our current meta-analysis.

This meta-analysis has detected the association between VDR gene polymorphism and DR in stratification analysis. However, there were still several limitations in this study. First, relatively limited number of case-control studies was included in meta-analysis. Thus, further studies involving large sample sizes are needed to archive more reliable conclusions on association between VDR gene polymorphisms and DR. Secondly, no analysis of combined genotype effects was done due to the small sample size. Third, the control groups within two eligible studies were including non-severe DR patients, which could lead to potential publication bias. Last but not least,, we did not include articles published in other languages only in English or Chinese. Although we have tried our best to review related articles in all databases, there were many unpublished, insufficient data studies missing.

\section{Conclusion}

This meta-analysis revealed a significant association between DR and VDR-FokI polymorphism in Chinese subjects, among included studies without publication bias, during a comparison analysis between normal subjects and DR patients, and among articles published after 2010. However, owing to the limitations of this current meta-analysis, further prospective studies with larger sample sizes are needed to confirm our findings.

\section{Acknowledgments}

Thanks to Heather in Shibo Foreign Language School for English editing and proof reading services.

Funding: This research was supported by grants from Liaoning Provincial Department of Education (No. LQNK201703) and Science and Technology Fund Project of Shenyang Medical College (No. 20171006).

\section{Conflict of interest}

None.

\section{References}

1. Cheung N, Mitchell P, Wong TY. Diabetic retinopathy. Lancet. 2010;376:124-136.

2. Resnikoff S, Pascolini D, Etya'ale D, Kocur I, Pararajasegaram R, Pokharel GP, et al. Global data on visual impairment in the year 2002. Bull World Health Organ. 2004;82:844-851.

3. Yau JW, Rogers SL, Kawasaki R, Lamoureux EL, Kow- alski JW, Bek T, et al. Global prevalence and major risk factors of diabetic retinopathy. Diabetes Care. 2012;35:556564.

4. Ting DS, Cheung GC, Wong TY. Diabetic retinopathy: global prevalence, major risk factors, screening practices and public health challenges: a review. Clin Exp Ophthalmol. 2016;44:260-277.

5. Liu L, Wu J, Yue S, Geng J, Lian J, Teng W, et al. Incidence Density and Risk Factors of Diabetic Retinopathy Within Type 2 Diabetes: A Five-Year Cohort Study in China (Report 1). Int J Environ Res Public Health. 2015;12:7899-7909.

6. Behl T, Kotwani A. Exploring the various aspects of the pathological role of vascular endothelial growth factor (VEGF) in diabetic retinopathy. Pharmacol Res. 2015;99:137-148.

7. Aydin E, Demir HD, Sahin S. Plasma and aqueous humor angiotensin-converting enzyme levels in patients with diabetic retinopathy. Curr Eye Res. 2010;35:230-234. 8. Obrosova IG, Kador PF. Aldose reductase/polyol inhibitors for diabetic retinopathy. Curr Pharm Biotechnol. 2011;12:373-385.

9. Ma K, Xu Y, Wang C, Li N, Li K, Zhang Y, et al. A cross talk between class A scavenger receptor and receptor for advanced glycation end-products contributes to diabetic retinopathy. Am J Physiol Endocrinol Metab. 2014;307:E1153-1165.

10. Gröber U, Kisters K. Influence of drugs on vitamin D and calcium metabolism. Dermatoendocrinol. 2012;4:158166.

11. Xu J, Li W, Ma J, Liu J, Sha H, Zhou S, et al. Vitamin D - pivotal nutraceutical in the regulation of cancer metastasis and angiogenesis. Curr Med Chem. 2013;20:41094120.

12. Consiglio M, Destefanis M, Morena D, Foglizzo V, Forneris M, Pescarmona G, et al. The vitamin D receptor inhibits the respiratory chain, contributing to the metabolic switch that is essential for cancer cell proliferation. PLoS One. 2014;9:e115816.

13. Alsalem JA, Patel D, Susarla R, Coca-Prados M, Bland $\mathrm{R}$, Walker EA, et al. Characterization of vitamin $\mathrm{D}$ production by human ocular barrier cells. Invest Ophthalmol Vis Sci. 2014;55:2140-2147.

14. Taverna MJ, Selam JL, Slama G. Association between a protein polymorphism in the start codon of the vitamin D receptor gene and severe diabetic retinopathy in C-peptide-negative type 1 diabetes. J. Clin. Endocrinol. Metabolomics. 2005;90:4803-4808. 
15. Cyganek K, Mirkiewicz-Sieradzka B, Malecki MT, Wolkow P, Skupien J, Bobrek J, et al. Clinical risk factors and the role of VDR gene polymorphisms in diabetic retinopathy in Polish type 2 diabetes patients. Acta Diabetologica. 2006;43:114-119.

16. Stang A. Critical evaluation of the Newcastle-Ottawa scale for the assessment of the quality of nonrandomized studies in meta-analyses. Eur J Epidemiol. 2010;25:603-605. 17. Moher D, Liberati A, Tetzlaff J, Altman DG, PRISMA Group. Preferred reporting items for systematic reviews and meta-analyses: the PRISMA statement. PLoS Med. 2009;6:e1000097.

18. Stroup DF, Berlin JA, Morton SC, Olkin I, Williamson GD, Rennie D, et al. Meta-analysis of observational studies in epidemiology: a proposal for reporting. Meta-analysis Of Observational Studies in Epidemiology (MOOSE) group. JAMA. 2000;283:2008-2012.

19. Wu YN, Zhang YP, Wang AP. Association Between DNA Polymorphism of Human Vitamin D Receptor(VDR) Gene and Type 2 Diabetic Retinopathy in Mongolian People. Inner Mongolia Medical Journal. 2010;42:271274.

20. Hou LJ, Huang DH, Han JJ. Relationship between Vitamin D receptor gene FokI polymorphism and diabetic retinopathy in China. Journal of Taishan Medical College. 2015;36:490-493.

21. Zhong X, Du Y, Lei Y, Liu N, Guo Y, Pan T. Effects of vitamin $\mathrm{D}$ receptor gene polymorphism and clinical characteristics on risk of diabetic retinopathy in Han Chinese type 2 diabetes patients. Gene. 2015;566:212-216.

22. Taverna MJ, Sola A, Guyot-Argenton C, Pacher N,
Bruzzo F, Slama G, et al. Taq I polymorphism of the vitamin $\mathrm{D}$ receptor and risk of severe diabetic retinopathy. Diabetologia. 2002;45:436-442.

23. Alcubierre N, Valls J, Rubinat E, Cao G, Esquerda A, Traveset A, et al. Vitamin D Deficiency Is Associated with the Presence and Severity of Diabetic Retinopathy in Type 2 Diabetes Mellitus. Journal of Diabetes Research. 2015; 2015:374178.

24. Hong YJ, Kang ES, Ji MJ, Choi HJ, Oh T, Koong SS, et al. Association between Bsm1 Polymorphism in Vitamin D Receptor Gene and Diabetic Retinopathy of Type 2 Diabetes in Korean Population. Endocrinol Metab (Seoul). 2015;30:469-474.

25. Bućan K, Ivanisević M, Zemunik T, Boraska V, Skrabić V, Vatavuk Z, et al. Retinopathy and nephropathy in type 1 diabetic patients--association with polymorphysms of vitamin D-receptor, TNF, Neuro-D and IL-1 receptor 1 genes. Coll Antropol. 2009;33:99-105.

26. Wrzosek M, Jakubczyk A, Wrzosek M, Kaleta B, Lukaszkiewicz J, Matsumoto $\mathrm{H}$, et al. Association between Fok I vitamin D receptor gene (VDR) polymorphism and impulsivity in alcohol-dependent patients. Mol Biol Rep. 2014;41:7223-7228.

27. Yu F, Cui LL, Li X, Wang CJ, Ba Y, Wang L, et al. The genetic polymorphisms in vitamin $\mathrm{D}$ receptor and the risk of type 2 diabetes mellitus: an updated meta-analysis. Asia Pac J Clin Nutr. 2016;25:614-624.

28. Zhang Y, Xia W, Lu P, Yuan H. The Association between VDR Gene Polymorphisms and Diabetic Retinopathy Susceptibility: A Systematic Review and Meta-Analysis. Biomed Res Int. 2016;2016:5305282. 